\title{
Interactions Between Tobamovirus Replication Proteins and Cellular Factors: Their Impacts on Virus Multiplication
}

\author{
Kazuhiro Ishibashi, Masaki Nishikiori, and Masayuki Ishikawa \\ Division of Plant Sciences, National Institute of Agrobiological Sciences, Tsukuba 305-8602, Japan
}

Submitted 30 April 2010. Accepted 30 June 2010.

\begin{abstract}
Most viral gene products function inside cells in the presence of various host proteins, nucleic acids, and lipids. Thus, viral gene products come into direct contact with these molecules. The replication proteins of tobamovirus participate not only in viral genome replication but also in counterdefense mechanisms against RNA silencing and other plant defense systems. Accumulating evidence indicates that these functions are carried out through interactions with specific host components. Interactions with some cellular factors, however, are inhibitory to virus multiplication and contribute to host range restriction of tobamovirus. The interactions that have positive and negative impacts on virus multiplication should have been maintained and lost, respectively, during adaptation of the viruses to their respective natural hosts. This review lists the host factors that interact with the replication proteins of tobamovirus and discusses how they influence multiplication of the virus.
\end{abstract}

All viral gene products are primarily synthesized in the cytoplasm by the host translation machinery, and most function inside the host cells. Therefore, virus-related molecules can make direct contact with numerous cellular components. Some of the interactions between viral and host factors are required for efficient viral multiplication or spread. Plant positivestrand RNA viruses Brome mosaic virus (BMV; genus Bromovirus) and Tomato bushy stunt virus (TBSV; genus Tombusvirus) can replicate in yeast Saccharomyces cerevisiae (Janda and Ahlquist 1993; Panavas and Nagy 2003). Genome-wide screening using the yeast single-gene-knockout library identified 58 genes whose deletion inhibited BMV RNA-mediated reporter gene expression by threefold or more, and 90 genes whose deletion inhibited accumulation of TBSV defectiveinterfering RNA by twofold or more (Kushner et al. 2003; Panavas et al. 2005). These results indicate that many host factors are required for efficient multiplication of BMV or TBSV RNA, although some of these factors may indirectly influence virus multiplication. Importantly, there are only three genes whose deletion impairs RNA replication of both BMV and TBSV, suggesting that viruses use different sets of host factors for multiplication (Panavas et al. 2005).

Not only do host organisms provide factors necessary for virus multiplication, they also defend themselves against viruses by RNA silencing and other resistance systems. Viruses often evolve counterdefense mechanisms to these host defense systems; for example, many viruses encode a suppressor of RNA

Corresponding author: M. Ishikawa; E-mail: ishika32@affrc.go.jp silencing with diverse structure and function (Díaz-Pendón and Ding 2008). Interactions between viral and host factors also include those important for elicitation or suppression of host defense responses. Theoretically, interactions maintained in natural hosts throughout the evolution of viruses should be beneficial to the survival of viruses.

The genus Tobamovirus is a group of plant positive-strand RNA viruses that has long served as a model to investigate the mechanisms of multiplication and interactions with host organisms (Scholthof 2004). The genome of a tobamovirus encodes at least four proteins (Goelet et al. 1982). After infection, the genomic RNA is translated in the cytoplasm of the host cell to produce proteins of approximately $130 \mathrm{kDa}(130 \mathrm{~K}$ protein) and its read-through product of $180 \mathrm{kDa}(180 \mathrm{~K}$ protein). These proteins harbor the methyltransferase (MT) domain that plays a role in $5^{\prime}$ capping of viral RNAs and the helicase (Hel) domain. The $180 \mathrm{~K}$ protein has the polymerase domain in the read-through region. The $130 \mathrm{~K}$ and $180 \mathrm{~K}$ proteins are collectively designated here as "replication proteins." In addition to its functions in RNA replication, the $130 \mathrm{~K}$ protein suppresses posttranscriptional gene silencing (PTGS) (Kubota et al. 2003; Ding et al. 2004). The replication proteins are also involved in viral cell-to-cell movement (Hirashima and Watanabe 2001). The other two tobamoviral proteins, the $30-\mathrm{kDa}$ cell-tocell movement protein and the coat protein, are encoded downstream $\left(3^{\prime}\right)$ of the replication proteins and are expressed from respective subgenomic mRNAs synthesized during viral RNA replication.

To date, more than 10 host proteins have been shown to interact with the replication proteins: most of these do so with the Hel domain (Table 1). In this review, we discuss the roles of these host factors in tobamovirus multiplication and pathogenicity.

Host factors that are recruited

for tobamovirus multiplication.

Tobamovirus multiplication 1. Tobamovirus multiplication 1 (TOM1) is a plant seven-pass transmembrane protein localized on both vacuolar membranes and unidentified membranes of higher density (Yamanaka et al. 2000; Hagiwara et al. 2003). In Arabidopsis thaliana, simultaneous knockout of TOM1 and its homolog TOM3 completely inhibits tobamovirus multiplication (Yamanaka et al. 2002, Fujisaki et al. 2006). In Nicotiana tabacum, simultaneous knockdown of the orthologs of TOM1 and TOM3 strongly inhibits tobamovirus multiplication (Asano et al. 2005), indicating that TOM1 and TOM3 are required for tobamovirus multiplication in plants.

TOM2A, a four-pass transmembrane protein, was also shown to be involved in tobamovirus multiplication. Knockout 
of the TOM2A gene and some of its homologs in A. thaliana partially inhibits the multiplication of a crucifer-infecting tobamovirus (Tobacco mosaic virus [TMV]-Cg) but has little effect on the multiplication of Tomato mosaic virus (ToMV; genus Tobamovirus) (Tsujimoto et al. 2003; Fujisaki et al. 2008). Thus, TOM2A is most likely not an essential factor of tobamovirus multiplication but functions as an enhancer of replication complex formation of some tobamoviruses. The results of a split ubiquitin-based two-hybrid assay suggested that TOM2A interacts with TOM1 and with itself (Tsujimoto et al. 2003).

Replication of all known eukaryotic positive-strand RNA viruses takes place on the host membrane (Mackenzie 2005; Dennison 2008). Although obvious amino acid sequences that function as membrane-targeting signals or membrane-spanning regions are not found in tobamovirus replication proteins, a fraction of the replication proteins binds tightly to the membranes of infected cells. Similar to other positive-strand RNA viruses, the tobamovirus RNA-synthesizing activity is bound to the membranes (Watanabe and Okada 1986; Young and Zaitlin 1986; Osman and Buck 1996). The subcellular fractionation pattern of the activity is quite similar not only to that of membrane-bound tobamovirus replication proteins but also to that of TOM1 (Hagiwara et al. 2003). In addition, a yeast SOS-recruitment assay suggested that TOM1 interacts with the Hel domain of tobamovirus replication proteins (Yamanaka et al. 2000, 2002). The binding was confirmed by copurification of TOM1 with the active tobamovirus replication proteins in detergent-solubilized membranes prepared from infected tobacco cells (Nishikiori et al. 2006). These observations are consistent with the suggestion that TOM1 is an integral component of tobamovirus replication complexes and helps tether tobamovirus replication proteins on membranes.

Consistent with the above suggestion, overexpression of TOM1 in tobacco leads to increased accumulation of the membrane-bound forms of the replication proteins and decreased accumulation of the soluble (nonmembrane-bound) ones. Despite increased amounts of membrane-bound replication proteins, overexpression of TOM1 inhibits tobamovirus multiplication, suggesting an important role for the soluble replication proteins (Hagiwara-Komoda et al. 2008). As described above, tobamovirus replication proteins participate in both viral RNA synthesis and suppression of PTGS. Agroinfiltration and other experiments demonstrated that TOM1 overexpression decreases the activity of tobamovirus replication proteins to suppress PTGS (Hagiwara-Komoda et al. 2008).

Another line of experiments also suggested a link between soluble replication proteins and PTGS suppression. A ToMV mutant, TLJ, which possesses an amino acid substitution
(C349Y) in the replication proteins, shows attenuated pathogenicity and reduced ability to suppress PTGS (Kubota et al. 2003). Hagiwara-Komoda and associates (2008) reported that the level of soluble replication protein accumulation in TLJ is lower than that in wild-type ToMV in infected cells. Thus, both TOM1 overexpression and TLJ mutation affect accumulation of the soluble replication proteins and the ability to suppress PTGS. Based on these results, it is likely that the soluble forms of replication proteins function in PTGS suppression (Hagiwara-Komoda et al. 2008).

Note that, although wild-type ToMV does not multiply efficiently in TOM1-overexpressing and -silenced plants, TLJ multiplies more efficiently in TOM1-silenced plants than in nontransgenic plants, perhaps due to increased levels of the soluble forms of the replication proteins (Hagiwara-Komoda et al. 2008). These results suggest that balanced accumulation of the soluble and membrane-bound replication proteins is important for efficient multiplication of tobamoviruses (Fig. 1). To achieve this balance, each tobamovirus may have evolved to have an optimal affinity of the replication proteins to TOM1 expressed in their respective natural hosts.

Eukaryotic translation elongation factor 1A. Eukaryotic translation elongation factor (eEF)1A binds to aminoacyltransfer RNAs and brings them to the A sites of translating ribosomes. Plant eEF1A interacts with the $3^{\prime}$ untranslated region of tobamovirus RNA as well as the MT domain of tobamovirus replication proteins (Zeenko et al. 2002; Yamaji et al. 2006). Knockdown of the $e E F 1 A$ genes results in reduced multiplication of tobamoviruses (Yamaji et al. 2010), suggesting the involvement of eEF1A in tobamovirus multiplication. Currently, however, the roles of eEF1A in tobamovirus RNA replication remain elusive.

Interactions of eEF1A or its homologs with the genomic RNA or replication-related proteins and the involvement of these proteins in viral RNA replication have also been reported for many other positive-strand RNA viruses, including bacteriophage $\mathrm{Q} \beta$, West Nile virus, Turnip yellow mosaic virus (TYMV; genus Tymovirus), and TBSV (Blumenthal and Carmichael 1979; Matsuda and Dreher 2004; Davis et al. 2007; Li et al. 2009). For bacteriophage Q $\beta$, EF-Tu, the bacterial homolog of eEF1A, is one of the four subunits of viral replicase. EF-Tu has been proposed to bind to $\mathrm{Q} \beta$ RNA, place its $3^{\prime}$ terminus at a proper position on the replicase, and facilitate initiation of negative-strand synthesis (Brown and Gold 1996).

General control derepressed 10. ToMV RNA polymerase purified from tomato leaves contains a protein that reacts with antibodies against wheat eukaryotic translation initiation factor (eIF) 3 and yeast general control derepressed (GCD) 10 (Osman and Buck 1997). GCD10 was initially believed to be one of the

Table 1. Cellular proteins that interact with tobamovirus replication proteins

\begin{tabular}{|c|c|c|c|c|}
\hline Protein & Domain $^{a}$ & Method $^{\text {b }}$ & Source & Reference \\
\hline AAA-ATPase & Hel & $\mathrm{Y} 2 \mathrm{H}$ & Tobacco & Abbink et al. 2002 \\
\hline ADRC & Hel & Y2H, far-Western & Tobacco & Shimizu et al. 2004 \\
\hline ATAF2 & Hel & Y2H, IP & Arabidopsis thaliana & Wang et al. 2009 \\
\hline eEF1a & MT & Y2H, IP & Tobacco & Yamaji et al. 2006 \\
\hline GCD10 & MT & $\mathrm{Y} 2 \mathrm{H}$ & Yeast & Taylor and Carr 2000 \\
\hline IAA26 & Hel & $\mathrm{Y} 2 \mathrm{H}$, dot-blot & A. thaliana, tomato & Padmanabhan et al. 2005, 2008 \\
\hline $\mathrm{N}$ & Hel & Y2H, IP & Nicotiana glutinosa & Ueda et al. 2006 \\
\hline NRIP1 & Hel & Y2H, IP & N. benthamiana & Caplan et al. 2008 \\
\hline Oxygen-evolving complex subunit & Hel & $\mathrm{Y} 2 \mathrm{H}$ & Tobacco & Abbink et al. 2002 \\
\hline $\mathrm{P} 58^{\mathrm{IPK}}$ & Hel & Y2H, IP & N. benthamiana, bovine & Bilgin et al. 2003 \\
\hline $\mathrm{Tm}-1 / \mathrm{tm}-1$ & $\mathrm{Hel}^{\mathrm{c}}$ & IP & Solanum habrochaites, tomato & Ishibashi et al. 2007, 2009 \\
\hline TOM1/TOM3 & Hel & $\mathrm{Y} 2 \mathrm{H}$ & A. thaliana & Yamanaka et al. 2000, 2002 \\
\hline 14-3-3 proteins & Hel & Far-Western & Tobacco & Konagaya et al. 2004 \\
\hline
\end{tabular}

${ }^{\mathrm{a}} \mathrm{Hel}=$ helicase and MT = methyltransferase.

${ }^{\text {b }} \mathrm{Y} 2 \mathrm{H}=$ yeast two-hybrid system and IP = immunoprecipitation.

${ }^{\mathrm{c}} \mathrm{K}$. Ishibashi and M. Ishikawa, unpublished result. 
eIF3 subunits but was later found to play a role in the modification of tRNA (Anderson et al. 1998). Antibodies to the yeast GCD10 protein inhibit exogenous template-dependent RNA synthesis by ToMV RNA polymerase, and the inhibition is reversed by prior addition of exogenous yeast GCD10 (Osman and Buck 1997). Taylor and Carr (2000) reported that yeast GCD10 interacts with the MT domain of ToMV replication proteins in a two-hybrid assay. Further investigations are needed to clarify the functions of GCD10/eIF3 subunits in tobamovirus RNA replication.

\section{Host factors that elicit or modulate host defense against tobamoviruses.}

ATAF2. Plants employ a basal defense system which is activated by pathogen infection. One of the key molecules in this basal defense response is salicylic acid (SA) (Vlot et al. 2009). Overexpression of the NAC domain transcription factor
ATAF2 of $A$. thaliana activates the expression of SA-induced defense genes, including $P R 1$, and ATAF2 knockout plants express the defense genes at low levels on SA treatment, indicating that ATAF2 is a positive regulator of the SA-dependent basal defense responses (Wang et al. 2009). ATAF2 was found to interact with the Hel domain of the TMV replication proteins (Wang et al. 2009). In response to TMV infection, expression of the ATAF2 mRNA is upregulated but the ATAF2 protein is destabilized in a proteasome-dependent manner (Wang et al. 2009). Thus, TMV may suppress SA-mediated host defense through degradation of ATAF2 triggered by the interaction with the replication proteins to promote systemic infection. How TMV replication proteins destabilize ATAF2 is an interesting question that remains to be answered.

$P 58^{I P K}$. In animals, virus infection activates double-stranded RNA-dependent protein kinase (PKR). PKR induces phosphorylation of the $\alpha$ subunit of eIF2 (eIF2 $\alpha)$, which results in

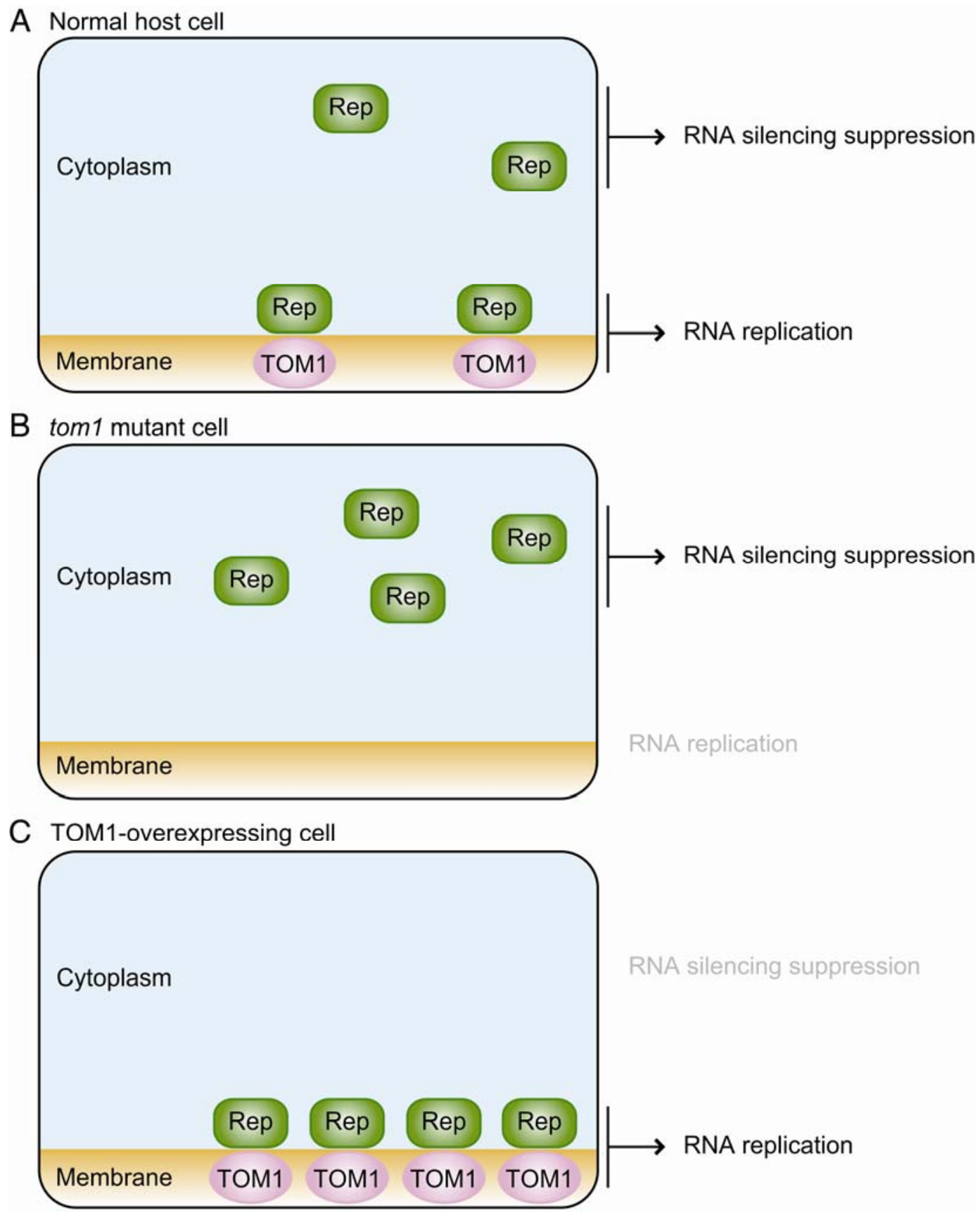

Fig. 1. Possible mechanism of the inhibition of tobamovirus multiplication by knockdown or overexpression of TOM1. 
translational suppression, activation of the type I interferon response, and apoptosis (Gil and Esteban 2000). P58 $8^{\mathrm{IPK}}$, a cellular inhibitor of PKR, is activated upon influenza virus infection via its dissociation from hsp40 (Lee et al. 1992; Melville et al. 1999). Activation of $\mathrm{P} 58^{\mathrm{IPK}}$ is thought to increase the production of influenza virus progeny by preventing PKR-mediated translational suppression and apoptosis (Goodman et al. 2009).

A plant ortholog of $\mathrm{P} 58^{\mathrm{IPK}}$ was found to interact with the Hel domain of TMV and Tobacco etch virus (TEV; genus Potyvirus) replication proteins (Bilgin et al. 2003), and the function of P58 $8^{\mathrm{IPK}}$ in plants has been investigated in depth. When $P 58^{I P K}$-silenced $N$. benthamiana plants are infected with TMV or TEV, or when $P 58^{I P K}$-knockout $A$. thaliana plants are infected with Turnip mosaic virus (genus Potyvirus) or Turnip vein clearing virus (genus Tobamovirus), severe cell death occurs (Bilgin et al. 2003). In $P 58^{I P K}$-silenced $N$. benthamiana plants, eIF $2 \alpha$ is phosphorylated upon TMV infection, and the expression of nonphosphorylatable eIF2 $\alpha$ mutant protein suppresses TMV-induced cell death (Bilgin et al. 2003). These results suggest that $\mathrm{P} 58^{\mathrm{IPK}}$ prevents cell death via inhibition of eIF2 $\alpha$ phosphorylation. As a consequence, TMV and TEV accumulate at lower levels in $P 58^{I P K}$-silenced $N$. benthamiana plants than in nonsilenced plants. Thus, TMV and TEV may activate $\mathrm{P} 58^{\mathrm{IPK}}$ similarly to influenza virus, possibly through the interaction with the replication proteins.

A PKR-like activity (i.e., a double-stranded RNA-dependent protein kinase activity that phosphorylates eIF $2 \alpha$ ) has been found in plants, which is upregulated upon infection of TMV or Potato spindle tuber viroid (Crum et al. 1988; Hiddinga et al. 1988; Langland et al. 1995, 1996; Chang et al. 1999). These results collectively suggest that the PKR pathway contributes to antiviral defense in plants. However, the genes responsible for the plant PKR-like activity have not yet been identified. Although A. thaliana GCN2 protein is known to phosphorylate eIF2 $\alpha$ (Lageix et al. 2008; Zhang et al. 2008), the relationship of this protein with the previously characterized PKR-like activity is still unclear.

Auxin/indole-3-acetic acid proteins. Auxin/indole-3-acetic acid (AUX/IAA) family proteins PAP1/IAA26, IAA27, and IAA 18 of $A$. thaliana and IAA26 of tomato interact with the Hel domain of TMV replication proteins (Padmanabhan et al. 2005, 2006, 2008). AUX/IAA family proteins are transcription factors that negatively regulate the expression of auxin-responsive genes. These proteins are localized in the nucleus and degraded at high auxin concentrations. In TMV-infected cells, these IAA proteins move from the nucleus to co-localize with the $130 \mathrm{~K}$ protein in the cytoplasm (Padmanabhan et al. 2006). This relocalization predicts the loss of IAA protein functions. Silencing of IAA26 causes the TMV-induced disease-like developmental abnormality in both $A$. thaliana and tomato, suggesting that the cytoplasmic sequestration of IAA26 by replication proteins accounts, at least in part, for the TMV-induced disease symptoms (Padmanabhan et al. 2005, 2008).

An amino acid substitution (V1087I) in the replication proteins of TMV disrupts the interaction with IAA26 (Padmanabhan et al. 2005). A TMV mutant that possesses a mutation causing this amino acid change normally multiplies in young leaves and shows mild disease symptoms (Padmanabhan et al. 2005, 2008). However, in old leaves in which IAA26 accumulates to higher levels than in young tissues, the mutant TMV multiplies less efficiently than wild-type TMV (Padmanabhan et al. 2008). In addition, overexpression of a proteolysis-resistant IAA26 mutant (P108L) protein results in reduced accumulation of TMV-V1087I, even in young leaves (Padmanabhan et al. 2008). Thus, AUX/IAA proteins are involved in age-related resistance, by which old plants and tissues are less susceptible to viruses; TMV replication proteins bind to and suppress the function of the AUX/IAA proteins to avoid the age-related resistance and, consequently, achieve high levels of multiplication. Padmanabhan and associates (2008) proposed that the symptoms associated with the loss of AUX/IAA functions are the inevitable result of overcoming the age-related resistance, because no evident advantage exists for virus survival gained from damaging the host.

$\mathrm{N}$ gene. In tobacco plants carrying the $N$ gene, a hypersensitive response that accompanies cell death takes place upon TMV infection to result in the inhibition of systemic spread of the virus (Holmes 1938). $N$ was bred into tobacco from the wild tobacco species $N$. glutinosa and encodes a nucleotide-binding site and leucine-rich repeat-containing protein (Whitham et al. 1994). The Hel domain of the TMV replication protein (p50) is the elicitor of the $N$-mediated resistance (Padgett et al. 1997; Abbink et al. 1998; Erickson et al. 1999). N protein interacts directly with p50 in an ATP-dependent manner (Ueda et al. 2006). In addition, tobacco 14-3-3 proteins and the chloroplastic protein NRIP1 bind both N and p50 (Konagaya et al. 2004; Caplan et al. 2008). NRIP1 is required for the resistance to $\mathrm{TMV}$, and relocalizes in the presence of p50 from the chloroplast to the cytoplasm and nucleus, where $\mathrm{N}$ exists (Caplan et al. 2008). More details regarding the action of $\mathrm{N}$ are discussed in another review in this issue (Padmanabhan and DineshKumar 2010).

\section{A cellular factor that binds to and inhibits the functioning of tobamovirus replication proteins.}

Tm-1/tm-1. Tm-1 is a semidominant trait of tomato (Solanum lycopersicum) that confers resistance to tobamoviruses (Porte et al. 1939; Pelham 1966; Fraser and Loughln 1980). It was originally introgressed from the wild tomato species $S$. habrochaites. Tm-1 prevents ToMV multiplication in protoplasts (Motoyoshi and Ohshima 1977) and, unlike most other disease resistance genes, does not induce a hypersensitive reaction (Yamafuji et al. 1991). Tm-1-breaking ToMV mutants have amino acid substitutions in the Hel domain of replication proteins, suggesting that Tm-1 recognizes ToMV replication proteins (Meshi et al. 1988; Hamamoto et al. 1997). Ishibashi and associates (2007) reported that the extract of $\mathrm{Tm}-1$ tomato cells inhibits ToMV RNA replication in vitro. The inhibitory activity was purified by fractionation of the cell extract to identify a protein responsible for the activity. The protein is composed of two uncharacterized domains and encoded by the $T m-1$ gene itself. Tm-1 binds to ToMV replication proteins but not to those of the resistance-breaking ToMV mutant, suggesting that binding of the Tm-1 protein causes inhibition of the functioning of ToMV replication proteins and that resistance-breaking mutants emerge by escaping from the inhibitory interaction (Ishibashi et al. 2007).

ToMV-susceptible tomato cultivars have a Tm-1 allele, $t m-1$. Although the amino acid sequence of the tm-1 protein shows $97 \%$ identity with that of Tm-1, tm-1 neither binds to ToMV replication proteins nor inhibits RNA replication (Ishibashi et al. 2007). Tobacco mild green mosaic virus (TMGMV) and Pepper mild mottle virus (PMMoV) are members of the genus Tobamovirus that can infect tobacco but not any cultivars of tomato (i.e., tomato is a nonhost of TMGMV and PMMoV). The multiplication of TMGMV and PMMoV is inhibited in transgenic tobacco expressing tm-1, and the tm- 1 protein binds to the replication proteins of TMGMV and PMMoV (Ishibashi et al. 2009). Thus, tm-1 is also an inhibitor of RNA replication and accounts, in part, for the inability of these tobamoviruses to multiply in tomato.

The homologs of the Tm-1 gene are conserved in many plants, fungi, archaea, and bacteria, suggesting that Tm- $1 / \mathrm{tm}-1$ has a primary although as-yet-undetermined function other than 
tobamoviral resistance and, incidentally, has the potential to bind to tobamovirus replication proteins and cause inhibition (Ishibashi et al. 2009). Several other findings (Zhu et al. 2007; Fujisaki and Ishikawa 2008; Mehle and Doudna 2008; Cheng et al. 2009; Li et al. 2009; Mahon et al. 2009; Sasaki et al. 2009) also suggest the presence of inhibitory factors for virus multiplication in organisms to which the virus is not well adapted. Thus, inhibitory interactions between viral and cellular factors may often occur in infected cells, although they are rarely found in the natural hosts because viruses must have escaped from such inhibitory factors. The lack of an interaction between the replication proteins of ToMV and tm-1 may represent such an evolutionary outcome.

\section{Concluding remarks.}

As described above, tobamovirus replication proteins interact with many cellular proteins. Although not discussed here due to the space limitation, they also interact with other host proteins (Abbink et al. 2002; Shimizu et al. 2004, Liu et al. 2005; Harris et al. 2010). In addition to interacting with host proteins, tobamovirus replication proteins interact with themselves (Goregaoker et al. 2001), with the tRNA-like structure of the viral genomic RNA (Osman and Buck 2003), and with small RNA duplexes (Mérai et al. 2006; Csorba et al. 2007; Kurihara et al. 2007; Hagiwara-Komoda et al. 2008).

Many interaction partners play distinct roles to facilitate virus multiplication. In such a situation, as TOM1 overexpression lowers the accumulation of soluble ToMV replication proteins that function as a PTGS suppressor, an excessive dominance of the binding of a particular host factor to the replication proteins can be inhibitory to overall virus multiplication because it may interfere with the function mediated by other interaction partners. Mutations in viral or host factors that increase the affinity to the partner can also lead to similar results, as found in the TLJ mutant case. Thus, to fulfill the multiple functions, tobamovirus replication proteins have likely evolved to have optimal (i.e., not too strong but not too weak) affinity to each interaction partner in their natural host cells that harbor given concentration of the host factors of given amino acid sequences.

On the other hand, some interactions that occurred in nonadapted organisms may be inhibitory, and indeed, tm-1 binds to TMGMV replication proteins to inhibit viral RNA replication in tomato. Such interactions should have been nullified in the natural hosts through adaptation and, thus, the ubiquitousness of inhibitory interactions may be underestimated. In addition to its role in host range restriction, negative interactions between viral and cellular factors may also play a role in avoidance of serious damage to the hosts due to overmultiplication (Mahajan et al. 2009).

\section{ACKNOWLEDGMENTS}

M. Ishikawa was funded by the Program for Promotion of Basic Research Activities for Innovative Biosciences; grant-in-aid for scientific research from the Ministry of Education, Culture, Sports, Science and Technology of Japan; and the Core Research for Evolutional Science and Technology, Japan Science and Technology Agency. M. Nishikiori is supported by a fellowship from the Japanese Society for the Promotion of Science.

\section{LITERATURE CITED}

Abbink, T. E. M., Tjernberg, P. A., Bol, J. F., and Linthorst, H. J. M. 1998. Tobacco mosaic virus helicase domain induces necrosis in $N$ gene-carrying tobacco in the absence of virus replication. Mol. Plant-Microbe Interact. 11:1242-1246.

Abbink, T. E. M., Peart, J. R., Mos, T. N., Baulcombe, D. C., Bol, J. F., and Linthorst, H. J. M. 2002. Silencing of a gene encoding a protein component of the oxygen-evolving complex of photosystem II en- hances virus replication in plants. Virology 295:307-319. 5.

Anderson, J., Phan, L., Cuesta, R., Carlson, B., Pak, M., Asano, K., Bjork, G., Tamame, M., and Hinnebusch, A. G. 1998. The essential Gcd10pGcd14p nuclear complex is required for 1-methyladenosine modification and maturation of initiator methionyl-tRNA. Genes Dev. 12:36503662 .

Asano, M., Satoh, R., Mochizuki, A., Tsuda, S., Yamanaka, T., Nishiguchi, M., Hirai, K., Meshi, T., Naito, S., and Ishikawa, M. 2005. Tobamovirus-resistant tobacco generated by RNA interference directed against host genes. FEBS (Fed. Eur. Biochem. Soc.) Lett. 579:4479-4484.

Bilgin, D. D., Liu, Y., Schiff, M., and Dinesh-Kumar, S. P. 2003. P58IPK, a plant ortholog of double-stranded RNA-dependent protein kinase PKR inhibitor, functions in viral pathogenesis. Dev. Cell 4:651-661.

Blumenthal, T., and Carmichael, G. G. 1979. RNA replication: Function and structure of Qbeta-replicase. Annu. Rev. Biochem. 48:525-548.

Brown, D., and Gold, L. 1996. RNA replication by Q $\beta$ replicase: A working model. Proc. Natl. Acad. Sci. U.S.A. 93:11558-11562.

Caplan, J. L., Mamillapalli, P., Burch-Smith, T. M., Czymmek, K., and Dinesh-Kumar, S. P. 2008. Chloroplastic protein NRIP1 mediates innate immune receptor recognition of a viral effector. Cell 132:449-462.

Chang, L. Y., Yang, W. Y., Browning, K., and Roth, D. A. 1999. Specific in vitro phosphorylation of plant eIF $2 \alpha$ by eukaryotic eIF $2 \alpha$ kinase. Plant Mol. Biol. 41:363-370.

Cheng, C.-W., Hsiao, Y.-Y., Wu, H.-C., Chuang, C.-M., Chen, J.-S., Tsai, C.-H., Hsu, Y.-H., Wu, Y.-C., Lee, C.-C., and Meng, M. 2009. Suppression of Bamboo mosaic virus accumulation by a putative methyltransferase in Nicotiana benthamiana. J. Virol. 83:5796-5805.

Crum, C. J., Hu, J., Hiddinga, H. J., and Roth, D. A. 1988. Tobacco mosaic virus infection stimulates the phosphorylation of a plant protein associated with double-stranded RNA-dependent protein kinase activity. J. Biol. Chem. 263:13440-13443.

Csorba, T., Bovi, A., Dalmay, T., and Burgyan, J. 2007. The p122 subunit of Tobacco mosaic virus replicase is a potent silencing suppressor and compromises both small interfering RNA- and microRNA-mediated pathways. J. Virol. 81:11768-11780.

Davis, W. G., Blackwell, J. L., Shi, P. Y., and Brinton, M. A. 2007. Interaction between the cellular protein eEF1A and the $3^{\prime}$-terminal stem-loop of West Nile virus genomic RNA facilitates viral minus-strand RNA synthesis. J. Virol. 81:10172-10187.

Denison, M. R. 2008. Seeking membranes: Positive-strand RNA virus replication complexes. PLoS Biol. 6:e270.

Díaz-Pendón, J. A., and Ding, S.-W. 2008. Direct and indirect roles of viral suppressors of RNA silencing in pathogenesis. Annu. Rev. Phytopathol. 46:303-326.

Ding, X. S., Liu, J., Cheng, N. H., Folimonov, A., Hou, Y. M., Bao, Y., Katagi, C., Carter, S. A., and Nelson, R. S. 2004. The Tobacco mosaic virus $126-\mathrm{kDa}$ protein associated with virus replication and movement suppresses RNA silencing. Mol. Plant-Microbe Interact. 17:583-592.

Erickson, F., Holzberg, S., Calderon-Urrea, A., Handley, V., Axtell, M., Corr, C., and Baker, B. 1999. The helicase domain of the TMV replicase proteins induces the $\mathrm{N}$-mediated defence response in tobacco. Plant J. 18:67-75.

Fraser, R. S. S., and Loughlin, S. A. R. 1980. Resistance to tobacco mosaic virus in tomato: Effect of the Tm-1 gene on virus multiplication. J. Gen. Virol. 48:87-96.

Fujisaki, K., and Ishikawa, M. 2008. Identification of an Arabidopsis thaliana protein that binds to Tomato mosaic virus genomic RNA and inhibits its multiplication. Virology 380:402-411.

Fujisaki, K., Ravelo, G. B., Naito, S., and Ishikawa, M. 2006. Involvement of THH1, an Arabidopsis thaliana homologue of the TOM1 gene, in tobamovirus multiplication. J. Gen. Virol. 87:2397-2401.

Fujisaki, K., Kobayashi, S., Tsujimoto, Y., Naito, S., and Ishikawa, M. 2008. Analysis of tobamovirus multiplication in Arabidopsis thaliana mutants defective in TOM2A homologues. J. Gen. Virol. 89:1519-1524.

Gil, J., and Esteban, M. 2000. Induction of apoptosis by the dsRNA-dependent protein kinase (PKR): Mechanism of action. Apoptosis 5:107114.

Goelet, P., Lomonossoff, G. P., Butler, P. J. G., Akam, M. E., Gait, M. J., and Karn, J. 1982. Nucleotide sequence of tobacco mosaic virus RNA. Proc. Natl. Acad. Sci. U.S.A. 79:5818-5822.

Goodman, A. G., Fornek, J. L., Medigeshi, G. R., Perrone, L. A., Peng, X., Dyer, M. D., Proll, S. C., Knoblaugh, S. E., Carter, V. S., Korth, M. J., Nelson, J. A., Tumpey, T. M., and Katze, M. G. 2009. P58IPK: A novel "CIHD" member of the host innate defense response against pathogenic virus infection. PLoS Pathog. 5:e1000438. Published online.

Goregaoker, S. P., Lewandowski, D. J., and Culver. J. N. 2001. Identification and functional analysis of an interaction between domains of the 126/183-kDa replicase-associated proteins of Tobacco mosaic virus. Virology 282:320-328.

Hagiwara, Y., Komoda, K., Yamanaka, T., Tamai, A., Meshi, T., Funada, 
R., Tsuchiya, T., Naito, S., and Ishikawa, M. 2003. Subcellular localization of host and viral proteins associated with tobamovirus RNA replication. EMBO (Eur. Mol. Biol. Organ.) J. 22:344-353.

Hagiwara-Komoda, Y., Hirai, K., Mochizuki, A., Nishiguchi, M., Meshi, T., and Ishikawa, M. 2008. Overexpression of a host factor TOM1 inhibits Tomato mosaic virus propagation and suppression of RNA silencing. Virology 376:132-139.

Hamamoto, H., Watanabe, Y., Kamada, H., and Okada, Y. 1997. Amino acid changes in the putative replicase of tomato mosaic tobamovirus that overcome resistance in Tm-1 tomato. J. Gen. Virol. 78:461-464.

Harries, P. A., Schoelz, J. E., and Nelson, R. S. 2010. Intracellular transport of viruses and their components: Utilizing the cytoskeleton and membrane highways. Mol. Plant-Microbe Interact. 23:1381-1393.

Hiddinga, H. J., Crum, C. J., and Roth, D. A. 1988. Viroid-induced phosphorylation of a host protein related to a dsRNA-dependent protein kinase. Science 241:451-453.

Hirashima, K., and Watanabe, Y. 2001. Tobamovirus replicase coding region is involved in cell-to-cell movement. J. Virol. 75:8831-8836.

Holmes, F. O. 1938. Inheritance of resistance to tobacco mosaic virus disease in tobacco. Phytopathology 28:553-561.

Ishibashi, K., Masuda, K., Naito, S., Meshi, T., and Ishikawa, M. 2007. An inhibitor of viral RNA replication is encoded by a plant resistance gene. Proc. Natl. Acad. Sci. U.S.A. 104:13833-13838.

Ishibashi, K., Naito, S., Meshi, T., and Ishikawa, M. 2009. An inhibitory interaction between viral and cellular proteins underlies the resistance of tomato to nonadapted tobamoviruses. Proc. Natl. Acad. Sci. U.S.A. 106:8778-8783.

Janda, M., and Ahlquist, P. 1993. RNA-dependent replication, transcription, and persistence of brome mosaic virus RNA replicons in S. cerevisiae. Cell 72:961-970.

Konagaya, K., Matsushita, Y., Kasahara, M., and Nyunoya, H. 2004. Members of 14-3-3 protein isoforms interacting with the resistance gene product $\mathrm{N}$ and the elicitor of Tobacco mosaic virus. J. Gen. Plant Pathol. 70:221-231.

Kubota, K., Tsuda, S., Tamai, A., and Meshi, T. 2003. Tomato mosaic virus replication protein suppresses virus-targeted posttranscriptional gene silencing. J. Virol. 77:11016-11026.

Kurihara, Y., Inaba, N., Kutsuna, N., Takeda, A., Tagami, Y., and Watanabe, Y. 2007. Binding of tobamovirus replication protein with small RNA duplexes. J. Gen. Virol. 88:2347-2352.

Kushner, D. B., Lindenbach, B. D., Grdzelishvili, V. Z., Noueiry, A. O., Paul, S. M., and Ahlquist, P. 2003. Systematic, genome-wide identification of host genes affecting replication of a positive-strand RNA virus. Proc. Natl. Acad. Sci. U.S.A. 100:15764-15769.

Lageix, S., Lanet, E., Pouch-Pélissier, M.-N., Espagnol, M.-C., Robaglia, C., Deragon, J.-M., and Pélissier, Thierry. 2008. Arabidopsis eIF $2 \alpha$ kinase GCN2 is essential for growth in stress conditions and is activated by wounding. BMC Pant Biol. 8:134.

Langland, J. O., Song, J., Jacobs, B., and Roth, D. A. 1995. Identification of a plant-encoded analog of PKR, the mammalian double-stranded RNA-dependent protein kinase. Plant Physiol. 108:1259-1267.

Langland, J. O., Langland, L. A., Browning, K. S., and Roth, D. A. 1996. Phosphorylation of plant eukaryotic initiation factor 2 by the plant encoded double-stranded RNA-dependent protein kinase, pPKR, and inhibition of protein synthesis in vitro. J. Biol. Chem. 271:4539-4544

Lee, T. G., Tomita, J., Hovanessian, A. G., and Katze, M. G. 1992. Characterization and regulation of the 58,000-dalton cellular inhibitor of the interferon-induced, dsRNA-activated protein kinase. J. Biol. Chem. 267:14238-14243.

Li, Z., Pogany, J., Panavas, T., Xu, K., Esposito, A. M., Kinzy, T. G., and Nagy, P. D. 2009. Translation elongation factor $1 \mathrm{~A}$ is a component of the tombusvirus replicase complex and affects the stability of the p33 replication co-factor. Virology 385:245-260.

Liu, J.-Z., Blancaflor, E. B., and Nelson, R. S. 2005. The Tobacco mosaic virus 126-kilodalton protein, a constituent of the virus replication complex, alone or within the complex aligns with and traffics along microfilaments. Plant Physiol. 138:1853-1865.

Mackenzie, J. 2005. Wrapping things up about virus RNA replication. Traffic 6:967-977.

Mahajan, V. S., Drake, A., and Chen, J. 2009. Virus-specific host miRNAs: Antiviral defenses or promoters of persistent infection? Trends Immunol. 30:1-7.

Mahon, C., Liang, B., Tikhanovich, I., Abend, J. R., Imperiale, M. J., Nasheuer, H. P., and Folk, W. R. 2009. Restriction of human polyomavirus BK virus DNA replication in murine cells and extracts. J. Virol. 83:5708-5717.

Matsuda, D., and Dreher, T. W. 2004. The tRNA-like structure of turnip yellow mosaic virus RNA is a $3^{\prime}$-translational enhancer. Virology 321:36-46.

Mehle, A., and Doudna. J. A. 2008. An inhibitory activity in human cells restricts the function of an avian-like influenza virus polymerase. Cell Host Microbe 4:111-122.

Melville, M. W., Tan, S.-L., Wambach, M., Song, J., Morimoto, R. I., and Katze, M. G. 1999. The cellular inhibitor of the PKR protein kinase, $\mathrm{P} 58 \mathrm{IPK}$, is an influenza virus-activated co-chaperone that modulates heat shock protein 70 activity. J. Biol. Chem. 274:3797-3803.

Mérai, Z., Kerenyi, Z., Kertesz, S., Magna, M., Lakatos, L., and Silhavy, D. 2006. Double-stranded RNA binding may be a general plant RNA viral strategy to suppress RNA silencing. J. Virol. 80:5747-5756.

Meshi, T., Motoyoshi, F., Adachi, A., Watanabe, Y., Takamatsu, N., and Okada, Y. 1988. Two concomitant base substitutions in the putative replicase genes of tobacco mosaic virus confer the ability to overcome the effects of a tomato resistance gene, Tm-1. EMBO (Eur. Mol. Biol. Organ.) J. 7:1575-1581.

Motoyoshi, F., and Oshima, N. 1977. Expression of genetically controlled resistance to tobacco mosaic virus infection in isolated tomato leaf mesophyll protoplasts. J. Gen. Virol. 34:499-506.

Nishikiori, M., Dohi, K., Mori, M., Meshi, T., Naito, S., and Ishikawa, M. 2006. Membrane-bound tomato mosaic virus replication proteins participate in RNA synthesis and are associated with host proteins in a pattern distinct from those that are not membrane bound. J. Virol. 80:84598468 .

Osman, T. A. M., and Buck, K. W. 1996. Complete replication in vitro of tobacco mosaic virus RNA by a template-dependent, membrane-bound RNA polymerase. J. Virol. 70:6227-6234.

Osman, T. A. M., and Buck, K. W. 1997. The tobacco mosaic virus RNA polymerase complex contains a plant protein related to the RNA-binding subunit of yeast eIF-3. J. Virol. 71:6075-6082.

Osman, T. A. M., and Buck, K. W. 2003. Identification of a region of the Tobacco mosaic virus 126- and 183-kilodalton replication proteins which binds specifically to the viral $3^{\prime}$-terminal tRNA-like structure. J. Virol. 77:8669-8675.

Padgett, H. S., Watanabe, Y., and Beachy, R. N. 1997. Identification of the TMV replicase sequence that activates the $\mathrm{N}$ gene-mediated hypersensitive response. Mol. Plant-Microbe Interact. 10:709-715.

Padmanabhan, M. S. and Dinesh-Kumar, S. P. 2010. All Hands on DeckThe Role of the Chloroplasts, Endoplasmic Reticulum, and Nucleus in Driving Plant Innate Immunity. Mol. Plant-Microbe Interact. 23:13681380 .

Padmanabhan, M. S., Goregaoker, S. P., Golem, S., Shiferaw, H., and Culver, J. N. 2005. Interaction of the Tobacco mosaic virus replicase protein with the Aux/IAA protein PAP1/IAA26 is associated with disease development. J. Virol. 79:2549-2558.

Padmanabhan, M. S., Shiferaw, H., and Culver, J. N. 2006. The Tobacco mosaic virus replicase protein disrupts the localization and function of interacting Aux/IAA proteins. Mol. Plant-Microbe Interact. 19:864-873.

Padmanabhan, M. S., Kramer, S. R., Wang, X., and Culver, J. N. 2008. Tobacco mosaic virus replicase-auxin/indole acetic acid protein interactions: Reprogramming the auxin response pathway to enhance virus infection. J. Virol. 82:2477-2485.

Panavas, T., and Nagy, P. D. 2003. Yeast as a model host to study replication and recombination of defective interfering RNA of Tomato bushy stunt virus. Virology 314:315-325.

Panavas, T., Serviene, E., Brasher, J., and Nagy, P. D. 2005. Yeast genomewide screen reveals dissimilar sets of host genes affecting replication of RNA viruses. Proc. Natl. Acad. Sci. U.S.A. 102:7326-7331.

Pelham, J. 1966. Resistance in tomato to tobacco mosaic virus. Euphytica 15:258-267.

Porte, W. S., Doolittle, S. P., and Wellman, F. L. 1939. Hybridization of a mosaic-tolerant, wilt-resistant Lycopersicon hirsutum with Lycopersicon esculentum. Phytopathology 29:757-759.

Sasaki, N., Ogata, T., Deguchi, M., Nagai, S., Tamai, A., Meshi, T., Kawakami, S., Watanabe, Y., Matsushita, Y., and Nyunoya, H. 2009. Over-expression of putative transcriptional coactivator KELP interferes with Tomato mosaic virus cell-to-cell movement. Mol. Plant Pathol. 10:161-173.

Scholthof, K.-B. G. 2004. Tobacco mosaic virus: A model system for plant biology. Annu. Rev. Phytopathol. 42:13-34.

Shimizu, T., Yamaji, Y., Ogasawara, Y., Hamada, K., Sakurai, K., Kobayashi, T., Watanabe, T., and Hibi, T. 2004. Interaction between the helicase domain of the Tobacco mosaic virus replicase and a tobacco arginine decarboxylase. J. Gen. Plant Pathol. 70:353-358.

Taylor, D. N., and Carr, J. P. 2000. The GCD10 subunit of yeast eIF-3 binds the methyltransferase-like domain of the 126 and $183 \mathrm{kDa}$ replicase proteins of Tobacco mosaic virus in the yeast two-hybrid system. J. Gen. Virol. 81:1587-1591.

Tsujimoto, Y., Numaga, T., Ohshima, K., Yano, M., Ohsawa, R., Goto, D. B., Naito, S., and Ishikawa, M. 2003. Arabidopsis TOBAMOVIRUS MULTIPLICATION (TOM) 2 locus encodes a transmembrane protein that interacts with TOM1. EMBO (Eur. Mol. Biol. Organ.) J. 22:335-343. 
Ueda, H., Yamaguchi, Y., and Sano, H. 2006. Direct interaction between the Tobacco mosaic virus helicase domain and the ATP-bound resistance protein, $\mathrm{N}$ factor during the hypersensitive response in tobacco plants. Plant Mol. Biol. 61:31-45.

Vlot, A. C., Dempsey, D. A., and Klessig, D. F. 2009. Salicylic acid, a multifaceted hormone to combat disease. Annu. Rev. Phytopathol. 47:177-206

Wang, X., Goregaoker, S. P., and Culver, J. N. 2009. Interaction of the Tobacco mosaic virus replicase protein with a NAC domain transcription factor is associated with the suppression of systemic host defenses. J. Virol. 83:9720-9730.

Watanabe, Y., and Okada, Y. 1986. In vitro viral RNA synthesis by a subcellular fraction of TMV-inoculated tobacco protoplasts. Virology 149:64-73.

Whitham, S., Dinesh-Kumar, S. P., Choi, D., Hehl, R., Corr, C., and Baker, B. 1994. The product of the tobacco mosaic virus resistance gene $N$ : Similarity to Toll and the interleukin-1 receptor. Cell 78:1101-1115.

Yamafuji, R., Watanabe, Y., Meshi, T., and Okada, Y. 1991. Replication of TMV-L and Lta 1 RNAs and their recombinants in TMV-resistant Tm-1 tomato protoplasts. Virology 183:99-105.

Yamaji, Y., Kobayashi, T., Hamada, K., Sakurai, K., Yoshii, A., Suzuki, M., Namba, S., and Hibi, T. 2006. In vivo interaction between Tobacco mosaic virus RNA-dependent RNA polymerase and host translation elongation factor 1A. Virology 347:100-108.
Yamaji, Y., Sakurai, K., Hamada, K., Komatsu, K., Ozeki, J., Yoshida, A., Yoshii, A., Shimizu, T., Namba, S., and Hibi, T. 2010. Significance of eukaryotic translation elongation factor $1 \mathrm{~A}$ in Tobacco mosaic virus infection. Arch. Virol. 155:263-268.

Yamanaka, T., Ohta, T., Takahashi, M., Meshi, T., Schmidt, R., Dean, C., Naito, S., and Ishikawa, M. 2000. TOM1, an Arabidopsis gene required for efficient multiplication of a tobamovirus, encodes a putative transmembrane protein. Proc. Natl. Acad. Sci. U.S.A. 97:10107-10112.

Yamanaka, T., Imai, T., Satoh, R., Kawashima, A., Takahashi, M., Tomita K., Kubota, K., Meshi, T., Naito, S., and Ishikawa, M. 2002. Complete inhibition of tobamovirus multiplication by simultaneous mutations in two homologous host genes. J. Virol. 76:2491-2497.

Young, N. D., and Zaitlin, M. 1986. An analysis of tobacco mosaic virus replicative structures synthesized in vitro. Plant Mol. Biol. 6:455-465.

Zeenko, V. V., Ryabova, L. A., Spirin, A. S., Rothnie, H. M., Hess, D., Browning, K. S., and Hohn, T. 2002. Eukaryotic elongation factor 1A interacts with the upstream pseudoknot domain in the $3^{\prime}$ untranslated region of Tobacco mosaic virus RNA. J. Virol. 76:5678-5691.

Zhang, Y., Wang, Y., Kanyuka, K., Parry, M. A. J., Powers, S. J., and Halford, N. G. 2008. GCN2-dependent phosphorylation of eukaryotic translation initiation factor-2 $\alpha$ in Arabidopsis. J. Exp. Bot. 59:3131-3141.

Zhu, J., Gopinath, K., Murali, A., Yi, G., Hayward, S. D., Zhu, H., and Kao, C. 2007. RNA-binding proteins that inhibit RNA virus infection. Proc. Natl. Acad. Sci. U.S.A. 104:3129-3134. 